\title{
Construction of Novel Coronavirus Pneumonia Emergency Management System Based on Multi Agent Cooperation Mechanism
}

\author{
JIN FENG SHANG* \\ Department of Director's Office, The Third People's Hospital of Jincheng, No.9, Chuandong Road, Jincheng, Shanxi 048000, \\ China
}

Shang et al.: Coronavirus Pneumonia Management System Based on Multi Agent Cooperation

In the prevention and control of the new crown pneumonia epidemic, the traditional emergency management system is restricted by the information exchange between multiple subjects, and in the actual process, its coordination is relatively poor. To this end, the establishment of an emergency management system for the prevention and control of new coronary pneumonia based on a multi agent cooperation mechanism is proposed. Establish different cooperation models between the subjects involved in the prevention and control of the new crown pneumonia epidemic, adjust the effectiveness of emergency tasks for the emergency tasks between different subjects, and build a prevention and control emergency management system based on the principle of the shortest time or the least resource utilization. Realize collaboration and cooperation between the various subjects. The experimental results show that the constructed emergency management system for the prevention and control of the new crown pneumonia epidemic based on a multi agent cooperation mechanism has less running time and high task completion in practical applications. Compared with the traditional emergency management system, its coordination is stronger.

Key words: Multi agent cooperation mechanism, novel coronavirus pneumonia, epidemic prevention and control, emergency management system

Although the new crown pneumonia epidemic has been under control, it has not yet completely resolved. Emergency management based on multiple entities has become a current research focus. The new crown pneumonia epidemic is a public emergency. A high level and effective emergency management system for public emergencies includes a complete organizational structure $^{[1]}$. In previous studies, due to the dynamics and complexity of emergencies, there are problems of decentralized departments, decentralized materials, and poor dynamic coordination among multiple entities. With the assistance of emergency management systems, some problems can be effectively solved ${ }^{[2]}$. However, the traditional emergency management system, in the face of the current outbreak of new crown pneumonia epidemic, which has a strong and wide range, involves more subjects in the management system, and is restricted by the information exchange between the subjects. In the actual application process, multiple subjects are involved. Coordination is extremely poor, and in the face of emergencies, it is difficult to play the role of coordination among multiple subjects ${ }^{[3-5]}$. Therefore, an emergency management system for the prevention and control of the new crown pneumonia epidemic with a multi agent cooperation mechanism is proposed to solve the problems existing in the above mentioned traditional emergency management system. In the novel coronavirus pneumonia epidemic prevention and control emergency management, the original social system structure is broken. The main body of emergency management is increasing gradually and the main body can only adapt to each other through information interaction between each other, and ultimately achieve a coordinated action ${ }^{[6]}$. With the increase of the number of subjects, the difficulty of coordination increases significantly, and the number of collaborative subjects is related to the type of emergency. In the face of unexplained emergencies, more subjects are involved, and coordination is more difficult ${ }^{[7]}$. Therefore, the cooperation mode based on multi agent cooperation mechanism is established in emergency management. When the relationship between the subjects is the 
upper and lower levels, the command execution mode is the main one. In this mode, the upper level issues clear instructions, and the lower levels execute them in accordance with the instructions, and apply them within the administrative system, which can effectively deal with emergencies event ${ }^{[8]}$. The instruction execution mode is not a one way mode. When the lower level finds a problem during the execution of the instruction, it will promptly report it to the higher level department and request decision support to help the higher level department make more accurate decisions ${ }^{[9]}$. In the face of emergencies under the new crown pneumonia epidemic, there are two organizational foundations for the instruction execution model, one is in the bureaucratic organization, and the other is in the command organization temporarily established during the emergency ${ }^{[10]}$. The novel coronavirus pneumonia outbreak is the fuse of emergencies. Only when we have a thorough understanding of the causes, transmission mode, diagnosis and treatment, we can alleviate the epidemic as soon as possible ${ }^{[1]}$. In this case, the assistant decision model can help the professional institutions and decision making institutions to carry out better collaboration. This mode is mainly used in the case of limited local professional strength, to establish this mode of cooperation between the central government departments and the local government. In the entire process of emergency management for the prevention and control of the new crown pneumonia epidemic, the instructions of the higher level government have clear action plans and principled content. In the actual management process, the local government needs to follow basic policies and continuously improve in practice. This requires innovation, support for learning mode. The innovation learning model mainly occurs between peer level organizations. In the prevention and control of the epidemic, different regions have different governance measures in the face of new coronary pneumonia. The innovation learning model helps different regions or different organizations to learn from each other. Situation, further strengthen the prevention and control of the new crown pneumonia epidemic, and improve the emergency management level of prevention and control in various regions ${ }^{[12]}$. The whole process of emergency management requires the overall arrangement of the decision making department, mobilize various social resources in the emergency process, and form a situation of multi-party cooperation in order to realize the efficient and reasonable allocation of emergency resources ${ }^{[13]}$. This requires the support of the overall planning cooperation model, which should be applied in the practice of emergency management to strengthen cooperation between the government and social organizations. Under the new crown pneumonia epidemic, in the face of emergencies, both financial support and human support are needed. Through the overall planning cooperation model, the deployment of human resources and material resources can be effectively coordinated, and the government can fully mobilize it under the overall planning and give full play to the enthusiasm and professionalism of all subjects, and improve the efficiency of cooperation among all subjects. Novel coronavirus pneumonia prevention and control emergency management requires not only the coordination between the government and social organizations, but also the coordination between the government and enterprises. The government and enterprises are the main body of the market transaction mode, and the market subject may be the victim of the epidemic situation or the main body to deal with the epidemic situation ${ }^{[14]}$. In the face of the epidemic, the government may not be able to directly assume the responsibility for the production of relevant materials or services. Under the market transaction mode, based on the transaction behavior, the government will cooperate with enterprises to support the consumption of various resources under the epidemic situation, and ensure people's basic life. The purpose of introducing efficiency adjustment into the prevention and control emergency management system is to dynamically adjust the emergency plan according to the situation of the event and the surrounding environment in the process of emergency management, so as to avoid the limitation of human and material resources due to the interactions between the main bodies. The novel coronavirus pneumonia outbreak may result in many kinds of results. $\mathrm{D}_{\mathrm{j}}=(\mathrm{j}=1,2, \ldots ., \mathrm{n})$ indicating the result of $j$, the probability of occurrence $p_{j}(j=1,2, \ldots \ldots, n)$, the plan for dealing with such events is $A=\left(A_{1}, A_{2}, \ldots . ., A_{m}\right)$, and different contingency plans have different disposal effects for each outcome. The emergency management adjustment project is shown in fig. 1 .

When dealing with emergency tasks, the previous emergency task may affect the next emergency task, which leads to differences in the difficulty of the adjustment of subsequent management work and the results of the emergency response. The adjustment efficiency is affected by the effect of the emergency plan implementation, capable of different disposal results in emergency management ${ }^{[15]}$. Suppose the emergency resources needed to adjust the incident to the best disposal plan after the occurrence of emergency is 
$\mathrm{u}$, and the adjustment time required is $\mathrm{t}$. The deviation between the disposal result of the adjusted scheme and the best effect is $\tau$, and the efficiency is expressed as $\mathrm{H}$. Then in the important mileage stage $\mathrm{B}_{\mathrm{i}}$ of emergency management, the adjustment efficiency of event result $\mathrm{D}_{\mathrm{j}}$ is as formula: $\mathrm{H}_{\mathrm{ij}}=\mathrm{H}_{\mathrm{ij}}\left(\mathrm{t}_{\mathrm{ij}}{ }^{(1)}, \mathrm{u}_{\mathrm{ij}}{ }^{(1)}, \tau_{\mathrm{ij}}\right)$. If the probability of occurrence of event result $D_{j}$ is $p_{j}$, then the expected value of adjustment effectiveness of $B_{i}$ is expressed as follows: $H_{i}=\Sigma p_{j} H_{i j}\left(t_{i j}{ }^{(1)}, u_{i j}{ }^{(1)}, \tau_{i j}\right)$. Generally, the result and time of performance adjustment is inversely proportional to the resources and deviation, that is, the smaller the resource and deviation, the better the effect of performance adjustment. Due to the different types of emergencies and other factors, the disposal effect is different. Assuming that the loss caused by the emergency is $\mathrm{k}$, the disposal efficiency $\mathrm{W}_{\mathrm{ij}}$ of the emergency plan adjusted according to result $D_{j}$ from $B_{i}$ is as follows: $W_{i j}=W_{i j}\left(t_{i j}{ }^{(1)}, u_{i j}{ }^{(1)}, k_{j}\right)$. $\mathrm{p}_{\mathrm{j}}$ is the probability of different disposal results, the expected disposal efficiency is expressed as follows: $\mathrm{W}_{\mathrm{ij}}=\sum_{i=1}^{n} p_{j} \mathrm{~W}_{\mathrm{ij}}\left(\mathrm{t}_{\mathrm{ij}}^{(1)}, \mathrm{u}_{\mathrm{ij}}{ }^{(1)}, \mathrm{k}_{\mathrm{j}}\right)$. By adjusting the efficiency of emergency tasks, it is ensured that under the emergency management system for the prevention and control of the new crown pneumonia epidemic, the management consumes the shortest time or uses the least resources. Under the support of multi agent cooperation mechanism, the prevention and control emergency management system is constructed, and the specific content is shown in fig. 2. It can be seen from fig. 2 that the constructed management system contains multiple entities. In the management process, the deployment and management of each entity is mainly based on a multi agent cooperation mechanism. According to the characteristics of the spread of new coronary pneumonia, under the situation of epidemic prevention and control, the consumption of materials is huge. In the face of emergencies, it is very likely that materials are limited and in short supply. Therefore, when materials are deployed, the efficiency of the above mentioned emergency tasks is adjusted, and the efficiency of the deployment of materials is adjusted with the goal of the least use of resources, to ensure the supply of materials. In the emergency situation of the epidemic, the deployment of human resources is also the top priority. Faced with the characteristics of the spread of the epidemic, the deployment of human resources must be completed in the shortest time while avoiding infection. In this deployment in the method, human resources are regarded as the main body, and with the support of a multi agent cooperation mechanism, the efficiency of human resource deployment tasks is adjusted to achieve the shortest time required for deployment, and to ensure that the entire deployment process is clear, orderly, strong in execution, and error free. For other subjects in the management system, the actual processing is the same as the above, all based on the principle of the shortest time and the least use of resources. In summary, the new crown pneumonia epidemic prevention and control emergency management system based on a multi agent cooperation mechanism can do a good job of prevention and control emergency management when fighting the

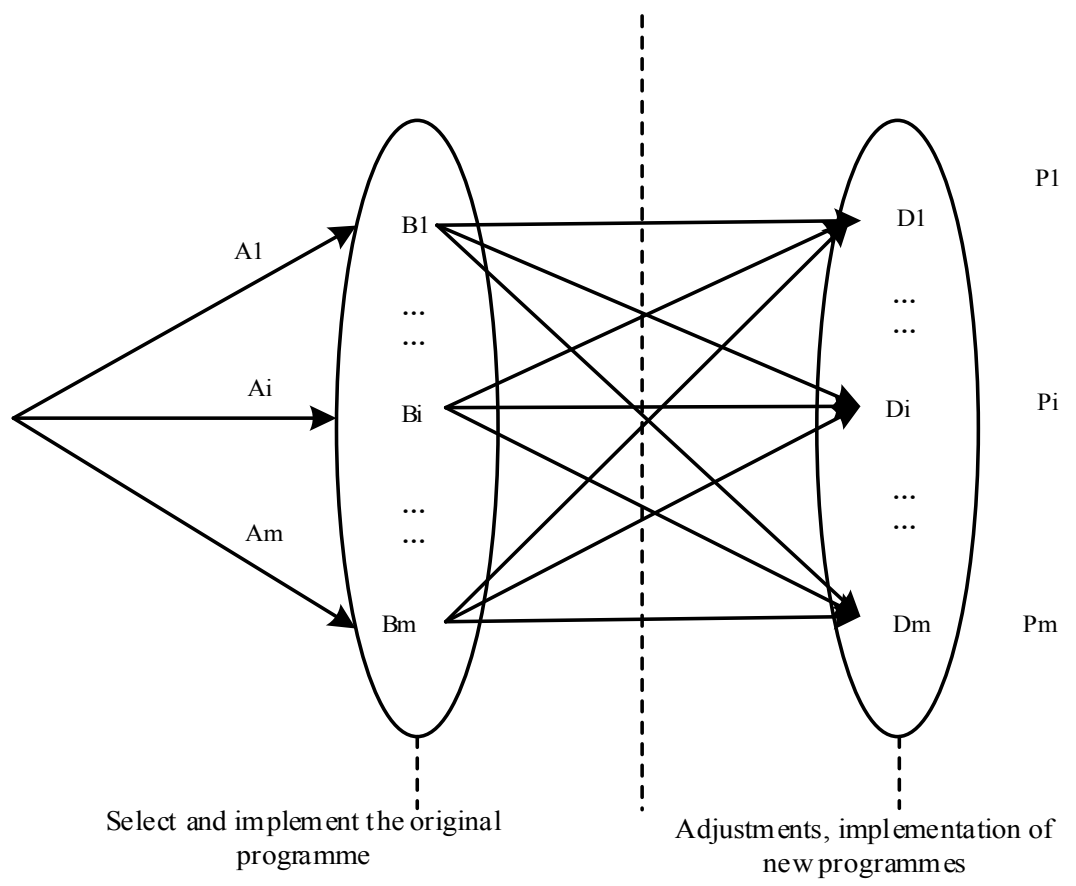

Fig. 1: Emergency management adjustment process 


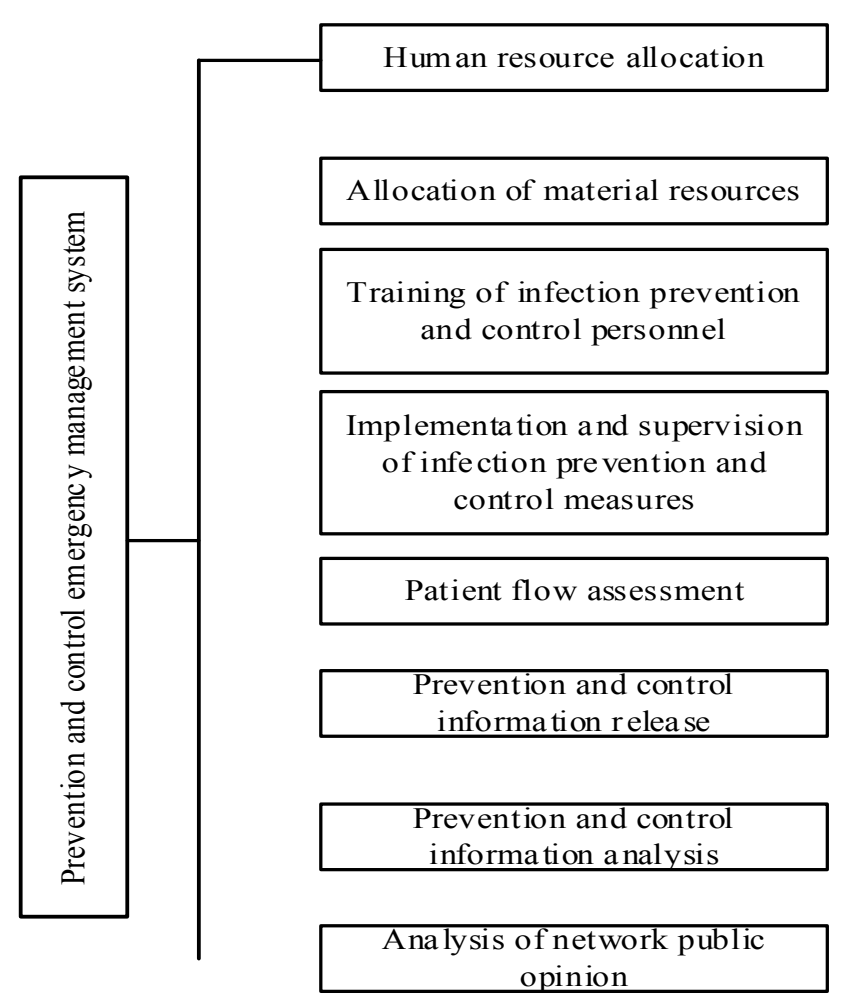

Fig. 2: Novel coronavirus pneumonia emergency management system

new crown pneumonia epidemic. In the experimental research on the prevention and control emergency management system, the cloud computing environment is simulated to verify the practical application level of the new crown pneumonia epidemic prevention and control emergency management system based on the multi agent cooperation mechanism. At the same time, a comparative experiment is designed to compare the proposed method with the traditional multi center The governed prevention and control emergency management system (method 1) and the prevention and control emergency management system based on digital technology (method 2) are compared under the same experimental environment, mainly to verify the coordination performance of different management systems for multiple tasks. Use the CloudSim tool set to simulate the cloud computing environment, set up execution tasks with different numbers of subjects, execute tasks under the action of different prevention and control emergency management systems, and use third party software to output experimental results. The specific results are shown in Table 1 and Table 2. The number shown in the table indicates the number of subjects involved in the execution of the task. Comparing the data in the observation table, it can be seen that the proposed method of prevention and control emergency management system faces an increase in the number of subjects in the task, and the time required to perform the task has a certain increase, but the increase is very small, and the completion of the task is $100 \%$. In contrast, with method 1 and method 2, as the number of task subjects increases, the time required to perform the task increases significantly, and the task completion degree decreases significantly. Method 1 is the most obvious, with multiple changes in data. In summary, the proposed method has better coordination when faced with multi agent tasks and the system is better than the traditional emergency management system for prevention and control. The novel coronavirus pneumonia has affected every single Chinese since its outbreak. To deal with such unexpected events, it is an important means to establish an emergency management system. The novel coronavirus pneumonia emergency management system is constructed and researched. Based on the support of large amount of research data and literature, a new crown pneumonia epidemic prevention and control emergency management system based on multi agent cooperation mechanism is designed. After the completion of the system, a cloud computing environment is established, and a comparative experiment is designed, which is in deep contrast with the traditional emergency management system. The experimental results show that the proposed emergency management system has high coordination, which verifies the reliability of the method in practical application meets the basic needs of emergency management in the face of emergency prevention and control at this stage and provides a reference for the setting of emergency plans. Novel coronavirus pneumonia is mainly considered in the current stage of the new crown pneumonia epidemic situation. In the face of different emergencies, there may be more

TABLE 1: TIME REQUIRED TO PERFORM TASKS UNDER DIFFERENT PREVENTION AND CONTROL EMERGENCY MANAGEMENT SYSTEMS (S)

\begin{tabular}{lccc}
\hline & Method 1 & Method 2 & The proposed method \\
\hline 3 & 3.94 & 5.45 & 1.19 \\
5 & 5.62 & 9.26 & 1.86 \\
9 & 11.25 & 14.26 & 2.16 \\
13 & 23.49 & 17.51 & 2.97 \\
15 & 29.35 & 23.14 & 3.28 \\
\hline
\end{tabular}

TABLE 2: TASK COMPLETION UNDER DIFFERENT PREVENTION AND CONTROL EMERGENCY MANAGEMENT SYSTEMS (\%)

\begin{tabular}{lccc}
\hline & Method 1 & Method 2 & The proposed method \\
\hline 3 & 98.6 & 99.6 & 100 \\
5 & 84.3 & 91.4 & 100 \\
9 & 77.1 & 87.2 & 100 \\
13 & 65.4 & 85.6 & 100 \\
15 & 63.6 & 83.2 & 100 \\
\hline
\end{tabular}


possibilities. In the follow up study, we will conduct in depth research on more different emergencies, expand the scope of use of the emergency management system and develop a higher level of emergency management system.

\section{Acknowledgements:}

This work was supported by The Third People's Hospital of Jincheng.

\section{Conflict of Interests:}

The authors declared no conflict of interest.

\section{REFERENCES}

1. Sciullo L, Trotta A, Di Felice M. Design and performance evaluation of a LoRa-based mobile emergency management system (LOCATE). Ad Hoc Netw 2020;96:101993.

2. Sun M, Xu N, Li C, Wu D, Zou J, Wang Y, et al. The public health emergency management system in China: trends from 2002 to 2012. BMC public health 2018;18:474.

3. Lei X, Zheng H, Shang Y, Wang H. Assessing emergency regulation technology in the middle route of the South-toNorth Water Diversion Project, China. Int J Water Resour Dev 2018;34:405-17.

4. Sanchez JN, Hudgens BR. Vaccination and monitoring strategies for epidemic prevention and detection in the Channel Island fox (Urocyon littoralis). PLoS One 2020;15:e0232705.

5. Soeters HM, Koivogui L, de Beer L, Johnson CY, Diaby D, Ouedraogo A, et al. Infection prevention and control training and capacity building during the Ebola epidemic in Guinea. PLoS One 2018;13:e193291.

6. Dobbs GC, Fogger SA. Opiate dependence or addiction: a review of the centers for disease control and prevention guidelines for management of chronic pain. J Addict Nurs 2018;29:57-61.

7. Malvy D, Gauzere BA, Migliani R. Epidemic and emerging prone-infectious diseases: Lessons learned and ways forward. Presse Med 2019;48:1536-50.

8. Anparasan AA, Lejeune MA. Data laboratory for supply chain response models during epidemic outbreaks. Ann Oper Res 2018;270:53-64.

9. Jacoby SF, Kollar LM, Ridgeway G, Sumner SA. Health system and law enforcement synergies for injury surveillance, control and prevention: a scoping review. Inj Prev 2018;24:305-11.

10. Zhang Y, Zhang X, Liu L, Wang H, Zhao Q. Suggestions for infection prevention and control in digestive endoscopy during current 2019-nCoV pneumonia outbreak in Wuhan, Hubei province, China. Endoscopy 2020;52:312-4.

11. Hsieh PJ, Lin WS. Understanding the performance impact of the epidemic prevention cloud: an integrative model of the task-technology fit and status quo bias. Behav Inf Technol 2020;39:899-916.

12. Li S, Liu Z, Li Y. Temporal and spatial evolution of online public sentiment on emergencies. Inf Process Manag 2020;57:102177.

13. Nolan K, Zullo AR, Bosco E, Marchese C, Berard-Collins C. Controlled substance diversion in health systems: A failure modes and effects analysis for prevention. Am J Health Syst Pharm 2019;76:1158-64.

14. Gachelin G, Garner P, Ferroni E, Verhave JP, Opinel A. Evidence and strategies for malaria prevention and control: a historical analysis. Malar J 2018;17:1-8.

15. Abubakar A, Bwire G, Azman AS, Bouhenia M, Deng LL, Wamala JF, et al. Cholera epidemic in South Sudan and Uganda and need for international collaboration in cholera control. Emerg Infect Dis 2018;24:883-7.

This is an open access article distributed under the terms of the Creative Commons Attribution-NonCommercial-ShareAlike 3.0 License, which allows others to remix, tweak, and build upon the work non-commercially, as long as the author is credited and the new creations are licensed under the identical terms

This article was originally published in a special issue, "Clinical Research in Pharmaceutical and Biomedical Sciences" Indian J Pharm Sci 2021:83(1)Spl issue1;106-110 